\title{
Stable Droplets and Growth Laws Close to the Modulational Instability of a Domain Wall
}

\author{
Damià Gomila, ${ }^{1,2}$ Pere Colet, ${ }^{1}$ Gian-Luca Oppo, ${ }^{2}$ and Maxi San Miguel ${ }^{1}$ \\ ${ }^{1}$ Instituto Mediterráneo de Estudios Avanzados, IMEDEA* (CSIC-UIB), Campus Universitat Illes Balears, \\ E-07071 Palma de Mallorca, Spain \\ ${ }^{2}$ Department of Physics and Applied Physics, University of Strathclyde, 107 Rottenrow, \\ Glasgow G4 ONG, Scotland, United Kingdom \\ (Received 31 May 2001; published 17 October 2001)
}

\begin{abstract}
We consider the curvature driven dynamics of a domain wall separating two equivalent states in systems displaying a modulational instability of a flat front. An amplitude equation for the dynamics of the curvature close to the bifurcation point from growing to shrinking circular droplets is derived. We predict the existence of stable droplets with a radius $R$ that diverges at the bifurcation point, where a curvature driven growth law $R(t) \approx t^{1 / 4}$ is obtained. Our general analytical predictions, which are valid for a wide variety of systems including models of nonlinear optical cavities and reaction-diffusion systems, are illustrated in the parametrically driven complex Ginzburg-Landau equation.
\end{abstract}

DOI: $10.1103 /$ PhysRevLett.87.194101

Growth of spatial domains of different phases in the transient regime of a system approaching thermodynamic equilibrium was studied long ago [1]. This phenomenon is a prototype of nonlinear dynamics of an extended system governed by the motion of domain walls. Power laws for the growth of a characteristic size and self-similar evolution (dynamical scaling) have been established. Clear physical mechanisms explaining the different asymptotic growth laws have also been identified [1]. It is convenient to consider domain growth in systems with conserved and nonconserved order parameters separately. In the first case, one talks of spinodal decomposition leading to the Lifshitz-Slyozov $t^{1 / 3}$ power law. In the second case, the dominant mechanism is curvature driven minimization of surface tension energy, leading to the Allen-Cahn (AC) $t^{1 / 2}$ power law [2].

The study of domain growth and domain wall motion in systems that do not approach thermodynamic equilibrium is by far more complicated. There is presently only a partial understanding of a variety of possible situations $[3,4]$. In particular a number of results have been recently reported for domain growth and domain wall motion in the transverse plane of nonlinear optical systems [5-9]. However, these numerical results often fail to identify the physical mechanism responsible for the observed dynamics. Moreover, the power laws for domain growth do not always correspond unambiguously to an asymptotic and self-similar dynamics. An exception is the $t^{1 / 2}$ law obtained for two different optical systems for which evidence of dynamical scaling has been given [6,7]. Evolutions of the output intensity profiles indicate curvature driven domain wall dynamics, but no derivation of such a law has been given for these systems. Surface tension energy, whose minimization may be used to explain the AC law, is not an appropriate concept for these systems.

In this Letter we analyze the transition from a regime characterized by a $t^{1 / 2}$ growth law to one of labyrinthine pattern formation due to a modulational instability of a flat
PACS numbers: $47.52 .+\mathrm{j}, 42.65 . \mathrm{Sf}, 47.20 . \mathrm{Ky}, 82.40 . \mathrm{Bj}$

domain wall connecting two equivalent homogeneous solutions. Such a transition from coarsening to labyrinthine regimes has been observed experimentally in reactiondiffusion [10] and optical [11] systems, and numerically in Ref. [6], as well as in Swift-Hohenberg models [12]. Differently from systems approaching thermodynamic equilibrium, the coefficient of the $t^{1 / 2}$ power law can now change sign upon variations of a control parameter $p$ at $p=p_{c}$. For $p<p_{c}\left(p>p_{c}\right)$ a circular domain of radius $R$ of one solution embedded in the other grows (shrinks as in the AC regime) as $R(t)=\sqrt{R(0)-\gamma t}$ with $\gamma<0(\gamma>0)$. Moreover, for $p<p_{c}$ a flat front is modulationally unstable and a generic initial condition develops into a labyrinthine pattern. Close to $p_{c}$ we derive an amplitude equation for the curvature of circular domains. At $p_{c}$ a circular domain grows as $R(t) \sim t^{1 / 4}$. For $p$ larger but close to $p_{c}$ an initially small (very large) domain grows (shrinks) until a stable droplet (SD) is formed. The radius of the SD diverges at $p_{c}$. The SD should not be confused with other types of solitonlike localized structures (LS) appearing in the AC regime when the oscillatory tails of the front prevent the domain collapse $[6,7,13]$. The SD are also different from the critical nuclei of nucleation theory [1], where the droplet is unstable and inner and outer solutions are not equivalent. Note also that droplets found in reaction-diffusion systems [14] involve two nonequivalent states.

Our analytical results are illustrated by considering a prototypical model, the parametrically driven complex Ginzburg-Landau equation (PCGLE) [15,16], which is the generic amplitude equation for an oscillatory system parametrically forced at twice its natural frequency [10]:

$$
\begin{aligned}
\partial_{t} A= & (1+i \alpha) \nabla^{2} A+(\mu+i \nu) A \\
& -(1+i \beta)|A|^{2} A+p A^{*},
\end{aligned}
$$

where $\mu$ measures the distance from the oscillatory instability threshold, $\nu$ is the detuning, and $p>0$ is the forcing 
amplitude. This model was used to describe a light sensitive form of the Belousov-Zhabotinsky reaction [10]. In nonlinear optics, models for vectorial Kerr cavities and optical parametric oscillators can be reduced to the PCGLE [17]. For $p \sim \nu \sim \alpha$ large compared to other parameters, a finite wavelength instability forming hexagons takes place for $p<p_{h}$, while for $p>p_{h}$ there are two equivalent stable homogeneous solutions (frequency locked solutions) [16].

We consider a real $N$ component vector field $\vec{\Psi}(\vec{x})$ whose dynamical evolution in two spatial dimensions is

$$
\partial_{t} \vec{\Psi}=D \nabla^{2} \vec{\Psi}+\vec{W}(\vec{\Psi}, p),
$$

where the matrix $D$ describes the spatial coupling, $\vec{W}$ is a local nonlinear function of the fields, and $p$ is a control parameter. Equation (2) is invariant under translations and under the change $\vec{x} \rightarrow-\vec{x}$. We also assume that it has a discrete symmetry $Z$ that allows for the existence of two, and only two, equivalent stable homogeneous solutions, and that, in a 1D system, they are connected by a stable Ising front $\vec{\Psi}_{0}(x, p)$. An Ising front satisfies $\vec{\Psi}_{0}\left(x_{0}-x\right)=Z \vec{\Psi}_{0}\left(x-x_{0}\right)$, where $x_{0}$ is the front location [18], thus the 1D front (and equivalently a flat front in 2D) is stationary, $D \nabla^{2} \vec{\Psi}_{0}+\vec{W}\left(\vec{\Psi}_{0}, p\right)=0$. For the PCGLE $\vec{\Psi}(\vec{x})=(\operatorname{Re}[A(\vec{x})], \operatorname{Im}[A(\vec{x})]), \quad D=$ $\left((1, \alpha)^{T},(-\alpha, 1)^{T}\right), \vec{\Psi}_{0}(x)$ describes a flat front connecting the two homogeneous solutions (we consider $p>p_{h}$ ) and $Z=-I$ where $I$ is the identity matrix.

Let $\vec{X}(s, t)$ represent the instantaneous position vector of the front in the $\vec{x}$ plane, where $s$ is the arclength. It is convenient to define a coordinate system $(r, s)$ that moves with the front such that $\vec{x}=\vec{X}(s, t)+r \hat{r}(s, t)$, where $\hat{r}$ is a unit vector normal to the curve $\vec{X}$, and the coordinate $r$ is the distance of the point $\vec{x}$ to the front [3]. In the moving frame Eq. (2) becomes

$$
\begin{aligned}
D \partial_{r}^{2} \vec{\Psi}+(v I & \left.+\frac{\kappa}{1+r \kappa} D\right) \partial_{r} \vec{\Psi}+ \\
& \frac{\kappa^{2} D \partial_{\theta}^{2} \vec{\Psi}}{(1+r \kappa)^{2}}+\vec{W}(\vec{\Psi}, p)=\partial_{t} \vec{\Psi},
\end{aligned}
$$

where $v=\partial_{t} \vec{X} \cdot \hat{r}$ is the (normal) front velocity, $\kappa=$ $\nabla \cdot \hat{r}$ is the curvature, and $\theta=\kappa s$ is the azimuthal angle. We analyze the dynamics of slightly curved fronts as a perturbation of the flat front $\vec{\Psi}(r, s, t)=$ $\vec{\Psi}_{0}(r)+\vec{\Psi}_{1}(r, s, t)$. We assume that (i) $\kappa w \ll 1$, with $w$ the front width, (ii) in the moving frame the front profile depends, at most, weakly on $t\left(\left|\partial_{t} \vec{\Psi}\right| \ll\left|\kappa D \partial_{r} \vec{\Psi}\right|\right)$, and (iii) $\kappa$ is a function which depends, at most, weakly on $s$, thus $\left|\kappa \partial_{\theta}^{2} \vec{\Psi}\right| \ll\left|\partial_{r} \vec{\Psi}\right|$. Linearizing around $\vec{\Psi}_{0}$ we have

$$
M \vec{\Psi}_{1}=-(v I+\kappa D) \partial_{r} \vec{\Psi}_{0},
$$

where $M_{j}^{i}=D_{j}^{i} \partial_{r}^{2}+\left.\delta_{\Psi^{j}} W^{i}\right|_{\Psi_{0}, p}$. Because of the translational invariance of (2) $M$ is singular, $M \vec{e}_{0}=0$ where $\vec{e}_{0} \equiv \partial_{r} \vec{\Psi}_{0}$ is the Goldstone mode. The solvability condition applied to (4) leads to $v=-\gamma(p) \kappa$, where

$$
\gamma(p) \equiv \frac{1}{\Gamma} \int_{-\infty}^{\infty} \vec{a}_{0} \cdot D \vec{e}_{0} d r,
$$

$\Gamma \equiv \int_{-\infty}^{\infty} \vec{a}_{0} \cdot \vec{e}_{0} d r$ [19], and $\vec{a}_{0}$ is the null mode of $M^{\dagger}$. For a circular domain, $\kappa=1 / R$ and

$$
v=\dot{R}=-\gamma(p) / R \text {. }
$$

From Eq. (4), one finds that the front perturbation $\vec{\Psi}_{1}(r, t)=\kappa(t) \vec{\varphi}_{1}(r)$ is independent of $s$ while the dependence on $t$ comes only through $\kappa \cdot \vec{\varphi}_{1}$ satisfies

$$
M \vec{\varphi}_{1}=-(-\gamma I+D) \vec{e}_{0} .
$$

For systems such that the diffusion matrix is proportional to the identity, $D=d I, \gamma$ takes the constant value $d$ independently of the profile of the front and any system parameter. In this case, the right-hand side of (7) vanishes and $\vec{\varphi}_{1}$ must be either zero or proportional to the Goldstone mode $\vec{e}_{0}$. Physically this means that the fronts translate without changing their radial profile. The front velocity is proportional to the curvature with opposite sign $v=-d \kappa$, which is the well-known AC law [1,2]. This law implies a coarsening regime with a $t^{1 / 2}$ growth law and shrinking of circular domains.

In general, $D=d I+C$ with a nonzero matrix $C$. $C$ leads to a contribution to $\gamma$ that depends on the profile of the front $\vec{\Psi}_{0}$ and, therefore, on the system parameters. From Eq. (7) we show that $\vec{\varphi}_{1}$ is no longer proportional to the Goldstone mode. This means that the transverse profile of the front is now deformed. Since $\vec{\Psi}_{1}(r, t)=\kappa(t) \vec{\varphi}_{1}(r)$, the amount of deformation is proportional to the curvature and $C$. The $d$ contribution is generally positive and, for wide parameter regions, $\gamma$ is also positive. Here flat walls are stable and the AC law still applies [6,7]. Circular domains shrink but the presence of oscillatory tails in the front may prevent the droplet from disappearing, forming a LS as found in nonlinear optical cavities $[6,7,13]$.

The crucial point is that for some parameter values the contribution to $\gamma$ due to $C$ may be negative and larger than $d$. $\gamma$ changes sign and a bifurcation occurs. This is particularly relevant in nonlinear optics where the spatial coupling is diffractive and $d=0$. For $\gamma<0$ the velocity has the same sign of the curvature leading to the growth of any perturbation of the flat wall. The condition for vanishing $\gamma, \int_{-\infty}^{\infty} \vec{a}_{0} \cdot D \vec{e}_{0} d r=0$, is in fact the criterion for the modulational instability of a flat front. Modulational instabilities in fronts connecting two equivalent homogeneous states have been observed in numerical models $[6,8]$. Starting from a random initial condition, the system develops labyrinthine patterns $[6,10]$. Also, a circular domain grows like (6) until its boundary breaks up because of the modulational instability leading to the formation of a labyrinthine pattern.

In Fig. 1 we show the value of $\gamma$ versus $p$ for the PCGLE calculated using definition (5). The 1D front profile 


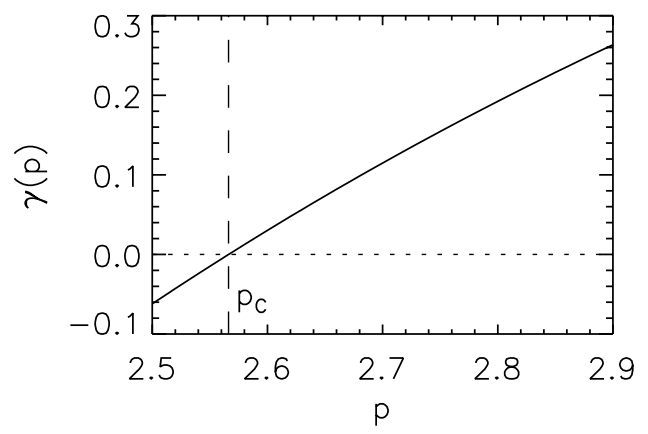

FIG. 1. $\quad \gamma$ as function of $p$ for the PCGLE calculated from (5). Here we take $\alpha=2, \beta=0, \nu=2$, and $\mu=0$. For these parameter values $p_{h}=2.09$ and $p_{c}=2.56629$ (dashed line).

was obtained by solving the stationary equation $\partial_{t} A=0$ in 1D of (1). The value of $p$ for which $\gamma=0$ is $p_{c}$.

We are now ready to obtain an amplitude equation for the curvature in the vicinity of $p_{c}$. We start by considering the case of a circular domain wall (for which $\left.v=-\dot{\kappa} / \kappa^{2}\right)$. Close to $p_{c}(\gamma \approx 0)$ we perform a multiple scale analysis in $\epsilon$ of Eq. (3) with $p=p_{c}+\epsilon p_{1}$, $\vec{\Psi}=\vec{\Psi}_{0}+\epsilon^{1 / 2} \vec{\Psi}_{1}+\epsilon \vec{\Psi}_{2}+\epsilon^{3 / 2} \vec{\Psi}_{3}, \kappa=\epsilon^{1 / 2} \kappa_{1}$, and $\partial_{t}=\epsilon^{2} \partial_{T}$. At order $\epsilon^{1 / 2}$ we obtain $M \vec{\Psi}_{1}=-\kappa_{1} D \vec{e}_{0}$. The solvability condition $\int_{-\infty}^{\infty} \vec{a}_{0} \cdot D \vec{e}_{0} d r=0$ is automatically satisfied at $p_{c}$. Then $\vec{\Psi}_{1}=\kappa_{1} \vec{\varphi}_{1}$ with $M \vec{\varphi}_{1}=$ $-D \vec{e}_{0}$. Because of the front symmetry the solvability condition at order $\epsilon$ is always fulfilled and $\vec{\Psi}_{2}=p_{1} \vec{\varphi}_{2}+$ $\kappa_{1}^{2} \vec{\varphi}_{3}$ with $M \vec{\varphi}_{2}=-\left.\partial_{p} \vec{W}\right|_{0}$ and $M_{j}^{i} \varphi_{3}^{j}=-D_{j}^{i}\left(\partial_{r} \varphi_{1}^{j}-\right.$ $\left.r e_{0}^{j}\right)-\left.\frac{1}{2} \delta_{\Psi^{j} \Psi^{k}} W^{i}\right|_{0} \varphi_{1}^{j} \varphi_{1}^{k}$, where $\left.\right|_{0}$ means being evaluated at $\vec{\Psi}_{0}$ and $p_{c}$. At order $\epsilon^{3 / 2}$ the amplitude equations for the curvature $\kappa_{1}$ and the radius of the circular domain,

$$
\begin{gathered}
\frac{\partial_{T} \kappa_{1}}{\kappa_{1}^{2}}=c_{1} p_{1} \kappa_{1}+c_{3} \kappa_{1}^{3}, \\
\partial_{t} R=-c_{1}\left(p-p_{c}\right) / R-c_{3} / R^{3},
\end{gathered}
$$

are obtained from the solvability condition with

$$
\begin{array}{r}
c_{1}=\frac{1}{\Gamma} \int_{-\infty}^{\infty} a_{0 i}\left(D_{j}^{i} \partial_{r} \varphi_{2}^{j}+\left.\delta_{\Psi^{j}} \partial_{p} W^{i}\right|_{0} \varphi_{1}^{j}\right. \\
\left.+\left.\delta \Psi^{j} \Psi^{k} W^{i}\right|_{0} \varphi_{1}^{j} \varphi_{2}^{k}\right) d r \\
c_{3}=\frac{1}{\Gamma} \int_{-\infty}^{\infty} a_{0 i}[ \\
D_{j}^{i}\left(\partial_{r} \varphi_{3}^{j}-r \partial_{r} \varphi_{1}^{j}+r^{2} e_{0}^{j}\right) \\
+\delta_{\left.\Psi^{j} \Psi^{k} W^{i}\right|_{0} \varphi_{1}^{j} \varphi_{3}^{k}} \\
\left.+\left.\delta \Psi^{j} \Psi^{k} \Psi^{l} W^{i}\right|_{0} \varphi_{1}^{j} \varphi_{1}^{k} \varphi_{1}^{l} / 6\right] d r .
\end{array}
$$

$c_{1}>0$ since $\gamma=c_{1}\left(p-p_{c}\right)$, and we are considering $\gamma>0$ for $p>p_{c}$. If $c_{3}$ is negative (supercritical bifurcation) our analysis predicts just above $p_{c}$ the existence of stable stationary circular domains (stable droplets) with a very large radius

$$
R_{0}=\frac{1}{\sqrt{p-p_{c}}} \sqrt{\frac{-c_{3}}{c_{1}}} .
$$

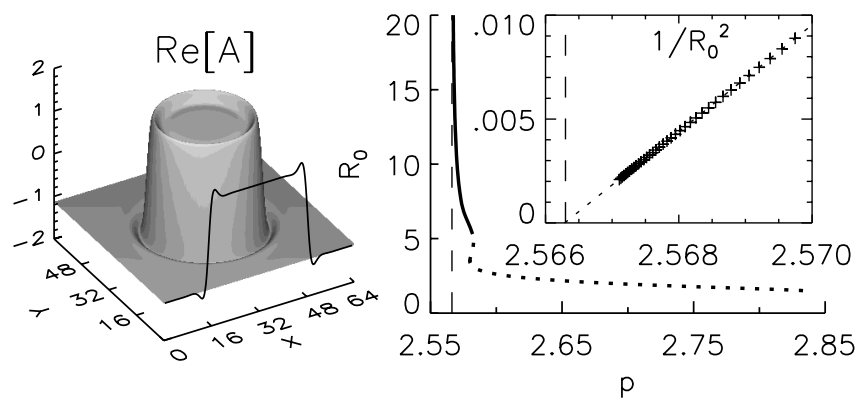

FIG. 2. Left: spatial dependence and transverse section of a SD for the PCGLE. Right: radius of SD (solid line) and LS (dotted line) as a function of the forcing for the PCGLE. The inset shows the linear dependence of $1 / R_{0}^{2}$ with $p$ close to the bifurcation point $p_{c}$ (dashed line) as predicted by (12).

In Fig. 2 (left) we show the form of the SD for the PCGLE. At the SD center the field closely approaches the value of one of the homogeneous solutions and the domain wall is close to a heteroclinic orbit between the two homogeneous states. The radius of the SD diverges to infinity at $p_{c}$. Figure 2 (right) displays the radius of the SD and that of the LS calculated by solving numerically $(1+i \alpha)\left(\partial_{r}^{2}+\right.$ $\left.\frac{1}{r} \partial_{r}\right) A+(\mu+i \nu) A-(1+i \beta)|A|^{2} A+p A^{*}=0$. The inset of Fig. 2 (right) shows the linear dependence of $1 / R_{0}^{2}$ with $p$ as predicted by (12). In spite of the fact that there is a smooth transition from LS to SD, they are intrinsically different. The oscillatory tail interaction, responsible for the existence of LS, does not play any role in the SD. The stabilization mechanism comes from the counterbalance between the $R^{-3}$ contribution to the front velocity and the shrinking due to the $R^{-1}$ contribution [20]. If $c_{3}>0$ (subcritical bifurcation) there would be unstable circular domains with radius $R_{0}$ just below $p_{c}$. However, we have never encountered this situation.

At $p_{c}$, Eq. (9) becomes $\partial_{t} R=-c_{3} / R^{3}$ and any circular domain of one solution embedded in the other grows as $R(t) \sim t^{1 / 4}$. In Fig. 3 we show the time evolution of the radius of a circular domain for the PCGLE at $p=p_{c}$.

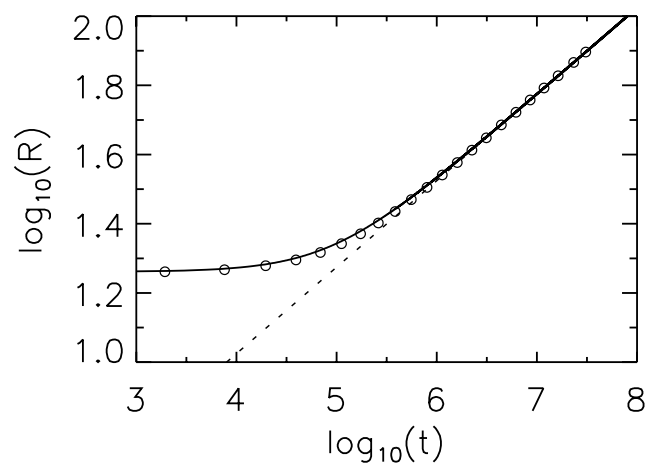

FIG. 3. Growth of a circular domain as a function of the time at $p=p_{c}$. The symbols correspond to the numerical integration of Eq. (1), the solid line is the theoretical prediction (9) with $c_{3}=0.3129$ calculated from (11), and the dotted line has a slope $1 / 4$ showing the asymptotic behavior. 

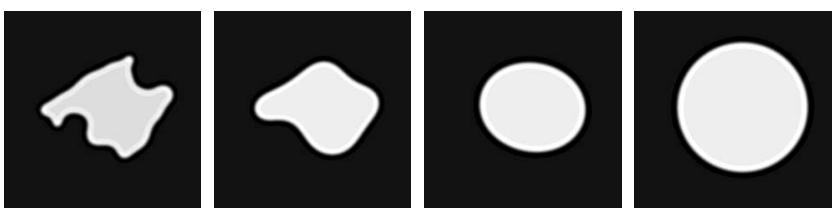

FIG. 4. Evolution of an arbitrarily shaped domain at $p=p_{c}$. From left to right: $t=0, t=2400, t=80000$, and $t=$ 1650000 .

It fits nicely with the theoretical dependence we predict. Close to $p_{c}$, in the regime of existence of the $\mathrm{SD}$, there is no asymptotic power law of domain growth since at very long times the SD is formed, stopping the growth process. During the transient, an initially small (very large) circular domain will grow (shrink) following (9).

So far we have considered the dynamics of domains with radial symmetry. When the system evolves from random initial conditions other dynamical mechanisms come into play. The main nonradially symmetric contribution to the velocity comes from the variation of the curvature along the front. The derivation of the amplitude equation for the curvature leads to an additional term in Eq. (8), $c_{2} \kappa_{1}^{2} \partial_{\theta}^{2} \kappa_{1}$, where $c_{2}=\frac{1}{\Gamma} \int_{-\infty}^{\infty} \vec{a}_{0} \cdot D \vec{\varphi}_{1} d r$. The front velocity becomes $v=-c_{1}\left(p-p_{c}\right) \kappa-c_{2} \kappa^{2} \partial_{\theta}^{2} \kappa-c_{3} \kappa^{3}$. Consistently with our approximations, $\partial_{\theta}^{2} \kappa$ will change at most at order $\kappa^{0}$, so the nonradial contribution is at least of order $\kappa^{2}$. At $p_{c}, c_{1}\left(p-p_{c}\right) \kappa$ vanishes, and the term given by $-c_{2} \kappa^{2} \partial_{\theta}^{2} \kappa$ may be dominant compared with $c_{3} \kappa^{3}$. The front velocity is then proportional to $\kappa^{2}$. However, for any closed boundary $\partial_{\theta}^{2} \kappa$ is positive in some parts of the wall and negative in others, so this term does not lead to an asymptotic growth law. For $c_{2}<0$, which is the case for the PCGLE, this term tends to reduce the curvature differences, so at $p_{c}$ an arbitrarily shaped domain first becomes circular until the contribution of $\partial_{\theta}^{2} \kappa$ vanishes and then the circular domain grows as $R(t) \sim t^{1 / 4}$ due to the $c_{3}$ term (see Fig. 4).

In summary, we have analyzed a generic situation of domain wall motion driven by curvature effects in which the proportionality coefficient $\gamma$ between wall velocity and curvature changes sign at a bifurcation point. In optical systems, this change is a consequence of the diffractive coupling between real and imaginary parts of the complex field amplitude. The amplitude equation for the curvature in the vicinity of the bifurcation point predicts the existence of stable nonlinear solutions which are droplets of one phase embedded in a background of the second equivalent phase. Nonlinear dynamics of the curvature leads to growth laws different from the AC $t^{1 / 2}$ growth law. The existence of a large characteristic length given by the radius of the SD destroys the possibility of self-similar evolution. We have observed these general results in nonlinear optical models for optical parametric oscillators with negative detuning and self-defocusing vectorial Kerr cavities with linearly polarized input field.
The authors acknowledge financial support from the EC TMR Network QSTRUCT (FMRXCT960077) and helpful discussions with G. K. Harkness and E. HernándezGarcía. Financial support from SGI and EPSRC (Grants No. M19727 and No. M31880) is acknowledged by G.L.O. and support from MCyT (Spain, Projects No. PB97-0141-C02-02 and No. BFM2000-1108) acknowledged by D. G., P.C., and M.S.M.

*Electronic address: http://www.imedea.uib.es/PhysDept

[1] J. D. Gunton, M. San Miguel, and P. Sahni, in Phase Transitions and Critical Phenomena, edited by C. Domb and J. Lebowitz (Academic Press, New York, 1983), Vol. 8, p. 269; A. Bray, Adv. Phys. 43, 357 (1994).

[2] S. M. Allen and J. W. Cahn, Acta Metall. 27, 1085 (1979).

[3] E. Meron, Phys. Rep. 218, 1 (1992).

[4] M.C. Cross and D. I. Meiron, Phys. Rev. Lett. 75, 2152 (1995); C. Josserand and S. Rica, ibid. 69, 1215 (1997); R. Gallego, M. San Miguel, and R. Toral, Phys. Rev. E 58, 3125 (1998).

[5] M. Tlidi, P. Mandel, and R. Lefever, Phys. Rev. Lett. 81, 979 (1998); M. Tlidi et al., Opt. Lett. 25, 487 (2000); M. Tlidi and P. Mandel, Europhys. Lett. 44, 449 (1998).

[6] R. Gallego, M. San Miguel, and R. Toral, Phys. Rev. E 61, 2241 (2000).

[7] G.-L. Oppo, A. J. Scroggie, and W. Firth, J. Opt. B Quantum Semiclassical Opt. 1, 133 (1999); Phys. Rev. E (to be published); G.-L. Oppo et al., J. Mod. Opt. 47, 2005 (2000).

[8] U. Peschel et al., Phys. Rev. E 58, R2745 (1998).

[9] M. Le Berre, E. Ressayre, and A. Tallet, J. Opt. B Quantum Semiclassical Opt. 2, 347 (2000).

[10] V. Petrov, Q. Ouyang, and H. L. Swinney, Nature (London) 388, 655 (1997).

[11] V. B. Taranenko, K. Staliunas, and C. O. Weiss, Phys. Rev. Lett. 81, 2236 (1998).

[12] K. Ouchi and H. Fujisaka, Phys. Rev. E 54, R3895 (1996); K. Staliunas and V. J. Sánchez-Morcillo, Phys. Lett. A 241, 28 (1998); V. J. Sánchez-Morcillo and K. Staliunas, Phys. Rev. E 60, 6153 (1999).

[13] M. Le Berre et al., J. Opt. B Quantum Semiclassical Opt. 1, 153 (1999); P. L. Ramazza et al., ibid. 2, 399 (2000).

[14] R. E. Goldstein, D. J. Muraki, and D. M. Petrich, Phys. Rev. E 53, 3933 (1996).

[15] P. Coullet et al., Phys. Rev. Lett. 65, 1352 (1990).

[16] P. Coullet and K. Emilsson, Physica (Amsterdam) 61D, 119 (1992).

[17] V. J. Sánchez-Morcillo et al., Opt. Lett. 25, 957 (2000); K. Staliunas, J. Mod. Opt. 42, 1261 (1995).

[18] D. Michaelis et al., Phys. Rev. E 63, 066602 (2001).

[19] $\Gamma$ vanishes at a Ising-Bloch transition [18]. Here we consider only parameter regions far away from any Ising-Bloch transition for which $\Gamma$ is never zero.

[20] A crossover between different growth laws has been found in systems with conserved order parameter [A. M. Lacasta et al., Phys. Rev. B 45, 5276 (1991)]. In that case, however, the coefficients have the same sign and there is no stationary radius for the domains. 